\title{
Emergence of novel phenomena on the border of low dimensional spin and charge order
}

\author{
Charles R.S. Haines ${ }^{1,2}$ and Siddharth S. Saxena ${ }^{1,3, a}$ \\ ${ }^{1}$ Cavendish Laboratory, Cambridge University, J.J. Thomson Ave, Cambridge CB3 0HE, UK \\ 2 Department of Earth Sciences, Cambridge University, Downing Street, Cambridge CB2 3EQ, UK \\ ${ }^{3}$ National University of Science and Technology "MISiS", Leninsky Prospekt 4, Moscow 119049, Russia
}

Received 30 May 2018 / Received in final form 5 June 2017

Published online 10 September 2018

(C) The Author(s) 2018. This article is published with open access at Springerlink.com, corrected publication October 2018

\begin{abstract}
Proximity to magnetic order as well as low dimensionality are both beneficial to superconductivity at elevated temperatures. Materials on the border of magnetism display a wide range of novel and potentially useful phenomena: high $T_{c} \mathrm{~s}$, heavy fermions, coexistence of magnetism and superconductivity and giant magnetoresistance. Low dimensionality is linked to enhanced fluctuations and, in the case of heavy fermions, has been experimentally shown to be beneficial for the fluctuations that are responsible for the rich abundance of novel emergent phases. This experimental strategy motivated us to explore 2D insulating magnets with a view to investigate phase evolution across metal-insulator and magnetic-non-magnetic boundaries. This has been a fruitful venture with totally novel results different to our expectations. We present results from 2 distinct systems. The $\mathrm{MPS}_{3}$ family are highly anisotropic in both their crystal and magnetic structures. $\mathrm{FePS}_{3}$ in particular is a model insulating honeycomb antiferromagnet. We find that the application of pressure to $\mathrm{FePS}_{3}$ induces an insulator to metal transition. The second system, $\mathrm{Cs}_{2} \mathrm{CuCl}_{4}$, is a highly-frustrated quantum spin liquid at low temperature. The competition of the 3 relevant exchange couplings is delicately balanced. It has been shown to become antiferromagnetic at very low temperatures $(\sim 1 \mathrm{~K})$. We have found that the application of pressure for 3 days or more followed by a return to ambient pressure stabilises a totally distinct magnetic ground state.
\end{abstract}

\section{Introduction}

A general feature of many of the most important discoveries in recent solid state physics and one that has been linked with a wide variety of novel states is the Mott insulator to metal transition. The most famous example is surely the high- $T_{c}$ cuprate superconductors. Additionally, iron based superconductivity has been associated with proximity to a Mott insulator state. In most of these systems the material is already a bad metal suggesting that it is close to the Mott insulator state but there is now growing evidence for systems in which the Mott insulator state can be realised [1].

The use of pressure is paritculary appealing as it affords control without the introduction of extra disorder. The Hubbard model can be used to describe both metallic and insulating states. However, the crossover from one to the other is not well described and the model is in general not solvable apart from the limiting cases. In addition

\footnotetext{
* Contribution to the Topical Issue "Coexistence of LongRange Orders in Low-dimensional Systems", edited by Sudhakar Yarlagadda and Peter B. Littlewood.

${ }^{a}$ e-mail: sss21@cam.ac.uk
}

to the metal insulator transition the suppression of magnetism is central to the understanding of the emergent novel states including superconductivity. $\mathrm{Cs}_{2} \mathrm{CuCl}_{4}$ has been suggested to be dominated by $1 \mathrm{D}$ and then $2 \mathrm{D}$ magnetic interactions. Frustration in this system leads to a quantum spin liquid state. Following the same philosophy of using pressure as a clean tuning parameter to investigate the emergence of novel phenomena in the vicinity of quantum phase transitions $\mathrm{Cs}_{2} \mathrm{CuCl}_{4}$ was pressurised. This was initially done with a view to a potential metallisation transition however, results published recently [2] show that even very modest pressure can have a profound effect on the magnetism in this material.

Whilst in many cases, and with some significant success, field tuning has been applied to low-dimensional magnets, the use of pressure to tune the underlying lattice and the exchange constants that it determines, is far less developed. On the other hand, pressure has been used to tune metallic systems, in particular narrow band f-electron metals like the heavy fermions. By employing the techniques from one field to examine the problems of the other we can hope to gain a new perspective. Pressure is used as the tuning parameter for $\mathrm{HF}$ and $\mathrm{QCP}$ as it can be thought of as tuning continuously from one (mostly) 


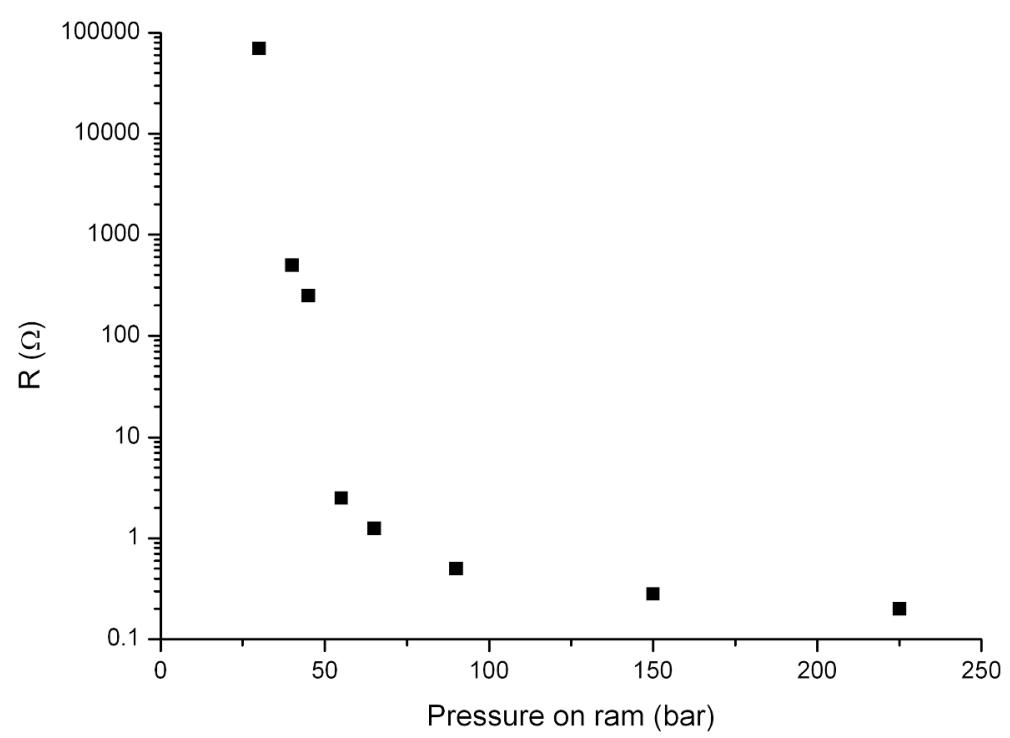

Fig. 1. The application of pressure results in a dramatic reduction of the room temperature resistivity.

known ground state to another, similarly to the effect of field-tuning on the low dimensional magnets. However, when applied to the balancing act of magnetic exchange in low dimensional frustrated insulating magnets, we do not necessarily know the ground state we will induce. We can therefore expect to be surprised. This cross-field learning will work both ways: the equivalent disruptive step in the study of the QCP seen in strongly correlated metal systems came when we discovered quantum criticality in insulating STO [3] and then subsequently the emergent low temperature state.

In these two cases we will present the use of moderate pressure has been used to change drastically the magnetic and electronic properties of the system. In both systems there is room for further work: we suggest that in $\mathrm{FePS}_{3}$ the metallic state is likely to be accompanied by a change in the magnetic state. Likewise, we expect that the new magnetic ground state of $\mathrm{Cs}_{2} \mathrm{CuCl}_{4}$ should also be related to new electronic properties.

\section{$2 \mathrm{FePS}_{3}$}

Substantial interest lies in tuning Mott insulators towards their metallisation transition, as the simple Hubbard model traditionally used to describe such systems only yields solutions in the limiting metallic and insulating cases - tuning the parameters of the system to an intermediate state accesses physics that are not yet fully understood. Additionally, many unconventional superconducting materials are low-dimensional and lie in close proximity to antiferromagnetic Mott insulator phases in their phase diagrams and theoretical calculations [4] suggest that these states have a strengthening influence on the formation of the superconductivity. Tunable (for instance through pressure) antiferromagnetic twodimensional Mott insulators then provide a rich and clean environment to probe the core mechanisms of several unsolved problems in condensed matter physics.
$\mathrm{FePS}_{3}$ is part of a family, $\mathrm{MPX}_{3}$, where $\mathrm{M}$ represents a transition metal such as $\mathrm{Fe}, \mathrm{Ni}$ or $\mathrm{Mn}$ and $\mathrm{X}=\mathrm{S}$ or Se. $\mathrm{MPX}_{3}$ have gone through various surges of interest. After they were first synthesised and the structures solved $[5,6]$ it was there potential for use in lithium batteries [5-15] that drove the research. A comprehensive review is given by Grasso and Silipigni [16]. Later, it was their diverse low dimensional magnetism that lead to a renewed interest. As the $a b$ planes of the metal ions are linked only by van-der-Waals forces, the $\mathrm{MPX}_{3}$ family form model two-dimensional antiferromagnetic systems, leading to a number of more recent publications on the magnetic structures [17-22]. These materials all have very similar crystal structures and interactions, but widely varying magnetic properties and structures due to the richness of the exchange couplings present. Single crystal X-ray diffraction studies $[5,6]$ give a monoclinic unit cell with space group of $\mathrm{C} 2 / \mathrm{m}$ and a honeycomb arrangement of the magnetic metal ions.

The extremely high room-temperature resistivities of the p-type semiconducting [16] $\mathrm{MPX}_{3}$ compounds reflect the high purity of samples that can be produced. The band gaps of the $\mathrm{MPX}_{3}$ compounds have been determined by optical measurements [23] and are in excess $1 \mathrm{eV}$. Early band structure calculations [14,24-26] were oversimplified and do not successfully reproduce the insulating state of these materials, predicting half-filled metallic bands. This leads to the conclusions that $\mathrm{MPX}_{3}$ are Mott insulators, and as such could be driven to an insulator-metal or Mott transition by applying pressure to tune the band structure. $\mathrm{FePS}_{3}$ has the lowest resistivity and band gap of the easily synthesised compounds and was therefore assumed to require the least pressure to metallise. Initial evidence of metallic behaviour was first reported in 2014 by Haines et al. [27] and then again independently by Tsurubayashi et al. in 2017 [28]. A recent article has combined high pressure structural data taken from XRD and high pressure resistivity data [29] to show the evolution of the electronic and crystal phases as a function of pressure. 

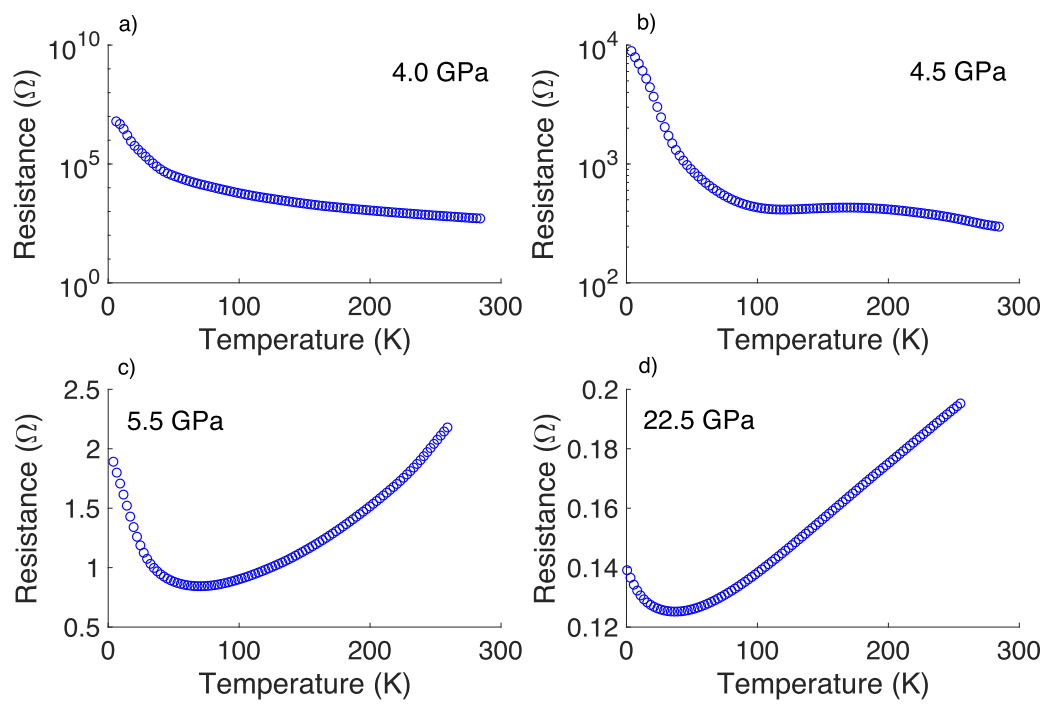

Fig. 2. Resistance of $\mathrm{FePS}_{3}$ against temperature for 4 increasing pressures, estimated as (a) $4.0 \mathrm{GPa}$, (b) $4.5 \mathrm{GPa}$, (c) $5.5 \mathrm{GPa}$ and (d) $22.5 \mathrm{GPa}$. A transition from insulating to metallic behaviour is seen as pressure is increased, as well as an upturn in the resistivity at low temperatures in the high pressure measurements. From [29].
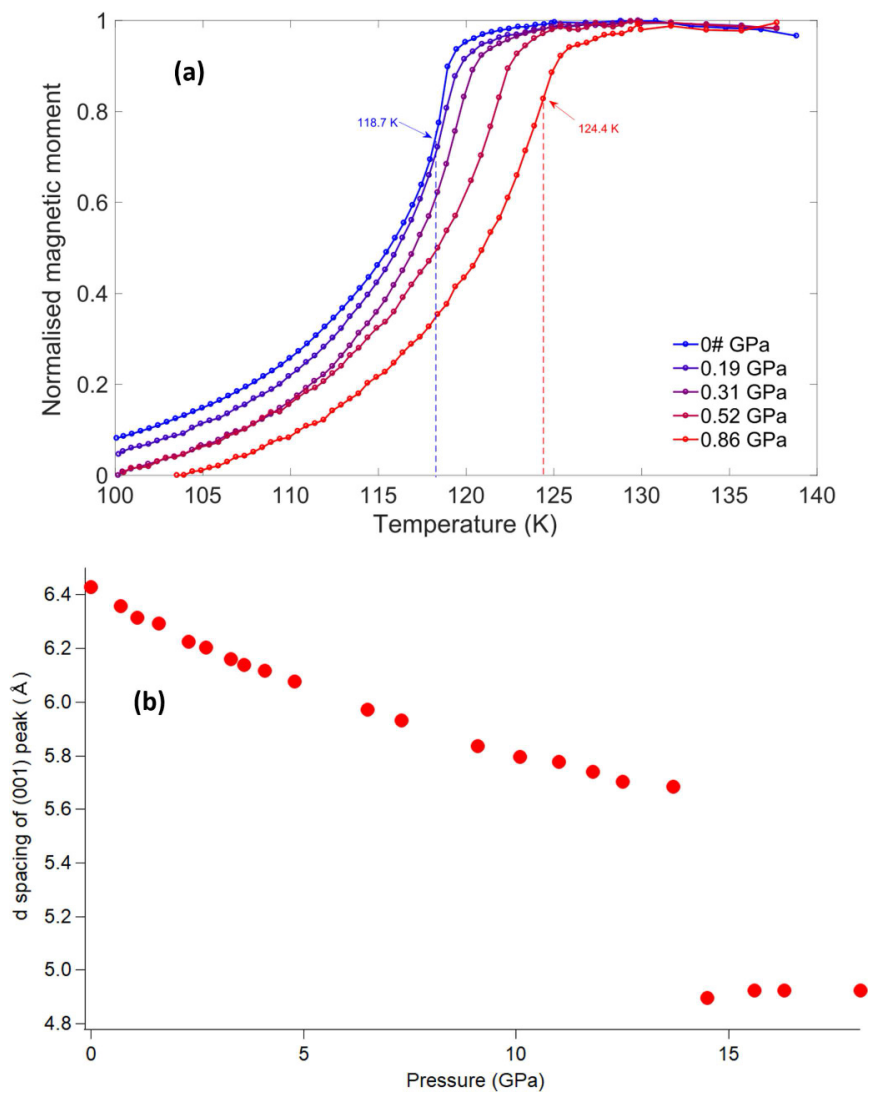

Fig. 3. Magnetisation of $\mathrm{FePS}_{3}$ plotted against temperature with increasing applied pressure. The interlayer spacing of $\mathrm{FePS}_{3}$ as a function of pressure. Until a major collapse at $14 \mathrm{GPa}$ pressure can be used to smoothly tune the interlayer spacing.

Initial measurements of the resistivity at room temperature as a function of pressure show a dramatic

but continuous evolution from high to low (Fig. 1). This was the first suggestion that the metallisation may be a continuous phase transition. Similar data (not shown) for $\mathrm{NiPS}_{3}$ is similar although the resistivity rises a little at the lowest pressures before reducing dramatically. Following on from this temperature sweeps at fixed pressures were measured (see Fig. 2). These measurements showed the resistivity to be smoothly varying with temperature as well as pressure even when the changes are very large.

Intriguingly there is an upturn in the resistivity at low temperatures in even the highest pressure data. It must be emphasised that this is seen in multiple samples and does not fit any exponential or modified exponential (i.e. variable range hopping). It is tempting to speculate that this may be attributable to some form of magnetic order; incipient or fully ordered.

The magnetism in $\mathrm{FePS}_{3}$ is important and my be critical in understanding the metallic state and the nature of the Metal-Insulator transition. Measuring anti-ferromagnetism under high pressure is a notoriously difficult problem. Using a miniaturised piston cylinder cell it was possible to see the initial effect of pressure. From XRD we see that the interlayer spacing is reduced smoothly by the application of pressure until the phase transition at $14 \mathrm{GPa}$. The magnetisation is dominated by the intralayer exchange. As pressure is applied the transition temperature initially increases. In other Mott insulators the AFM is suppressed rapidly as the system is tuned towards the metallisation transition. Following the Neel temperature at higher applied pressures through magnetometery is difficult as the feature in the susceptibility is relatively small and the sample size gets much reduced in anvil cells. High pressure neutron diffraction is a promising direction.

The same XRD data reveal two phase transitions. The first is at $4 \mathrm{GPa}$ and from Figure $3 \mathrm{~b}$ it is clear that there is no $c$-axis collapse associated with this transition. The 

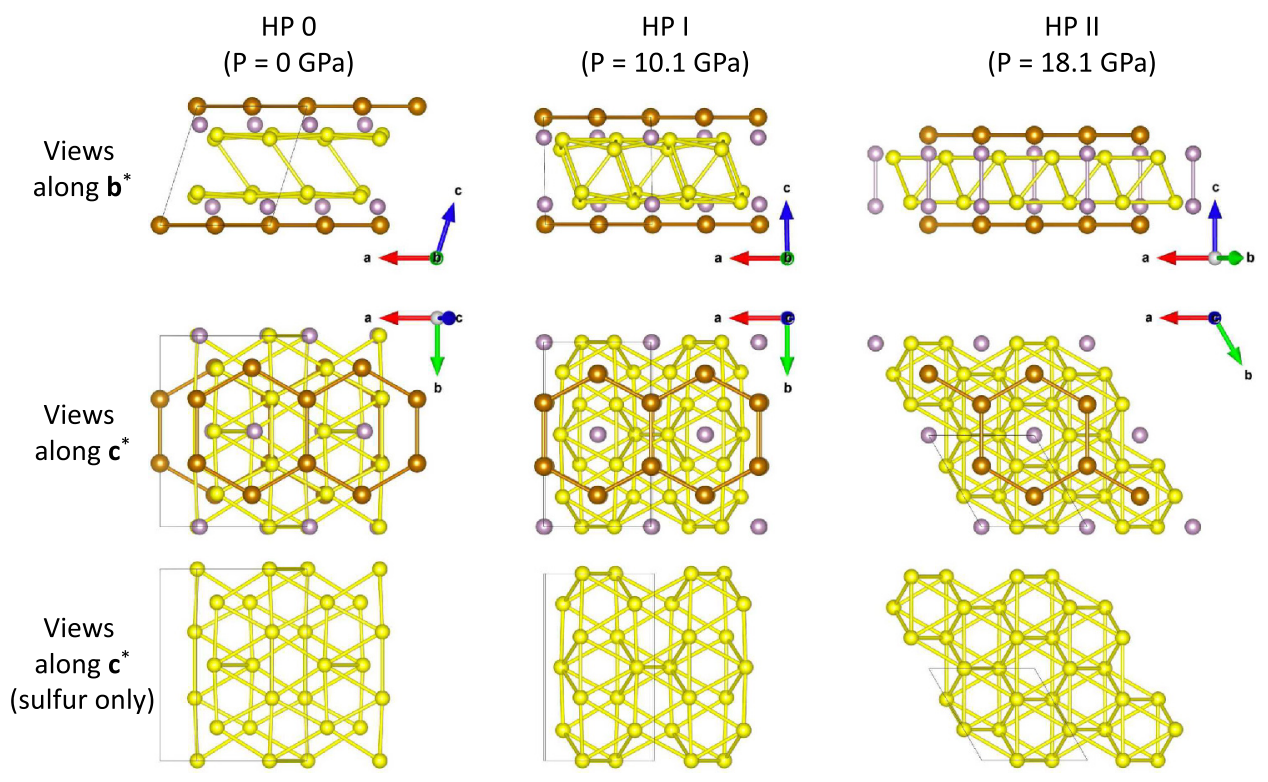

Fig. 4. Schematics showing the evolution of the structure of $\mathrm{FePS}_{3}$ with pressure. The three refined structures at their corresponding pressures are drawn to scale. The Fe atoms are shown in brown, the $\mathrm{P}$ atoms are shown in purple, and the $\mathrm{S}$ atoms are shown in yellow. The views show different projections of the same number of unit cells, hence the "sulfur only" figures show only those sulfurs between two adjacent ab planes. Also shown are all interatomic bonds for $r \leq 3.6 \AA$. The figures were created using the VESTA software [30]. From [29].

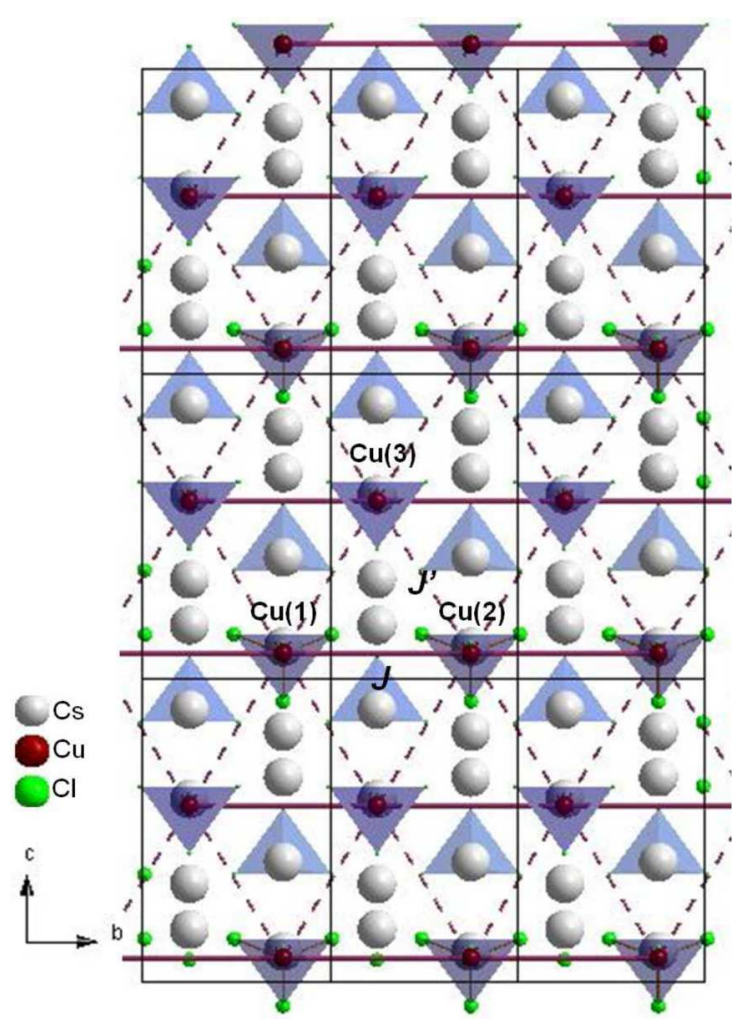

Fig. 5. Crystal structure of $\mathrm{Cs}_{2} \mathrm{CuCl}_{4}$. Orthorhombic crystal structure for the frustrated quantum antiferromagnet spin-1/2 $\mathrm{Cs}_{2} \mathrm{CuCl}_{4}$ with $\mathrm{Cu}^{2+}$ surrounded by $\mathrm{Cl}^{-}$ions and $\mathrm{Cs}^{+}$. The two antiferromagnetic spin interactions are the nearest neighbour exchange coupling $\mathrm{J}$ between $\mathrm{Cu}(1)$ and $\mathrm{Cu}(2)$ in the b-direction and the next nearest neighbour exchange coupling $\mathrm{J}$ between $\mathrm{Cu}(1)$ and $\mathrm{Cu}(3)$ on the triangular bc plane with a ratio of $\mathrm{J} / \mathrm{J}=0.34$. From [2]. metallic state in $\mathrm{FePS}_{3}$ is seen in resistivity measurements at approximately the same pressure of $4 \mathrm{GPa}$. Intriguingly this transition seems to involve no change of symmetry. It is tempting then to speculate that this transition may be driven by the electronic structure and could then be the Mott insulator to metal transition we set out to find. It is also possible that the metallisation takes place at the second higher pressure structural phase transition at which the $c$-axis (and the cell volume) collapse dramatically. The $4 \mathrm{GPa}$ transition can be thought of as a shearing of the monolayers along the $a$-axis (Fig. 4). It is not clear whether this is a sudden transition, however it does not increase the symmetry of the lattice.

\section{$3 \mathrm{Cs}_{2} \mathrm{CuCl}_{4}$}

As we have seen from $\mathrm{FePS}_{3}$ the potential for novel states in low dimensional magnetic insulators is great. $\mathrm{Cs}_{2} \mathrm{CuCl}_{4}$ has finely balanced magnetic exchange. Exactly as above then, we can image that if $\mathrm{Cs}_{2} \mathrm{CuCl}_{4}$ can be metallised the resulting metallic state is very likely to be highly correlated including the presence of long range magnetic order or significant fluctuations. Band structure calculations have concluded that $\mathrm{Cs}_{2} \mathrm{CuCl}_{4}$ is a Mott insulator [30].

Below $2.8 \mathrm{~K} \mathrm{Cs}_{2} \mathrm{CuCl}_{4}$ is a quantum spin liquid phase with antiferromagnetic interaction. In recent work we have reported the observation of a new metastable magnetic phase of $\mathrm{Cs}_{2} \mathrm{CuCl}_{4}$. Unusually, this phase is only stable after the application of hydrostatic pressure for more than 3 days. The magnetic properties of $\mathrm{Cs}_{2} \mathrm{CuCl}_{4}$ following the application and release of pressure after 3 days are strikingly different to those of the spin liquid state: we observed a previously unknown ordered magnetic phase 

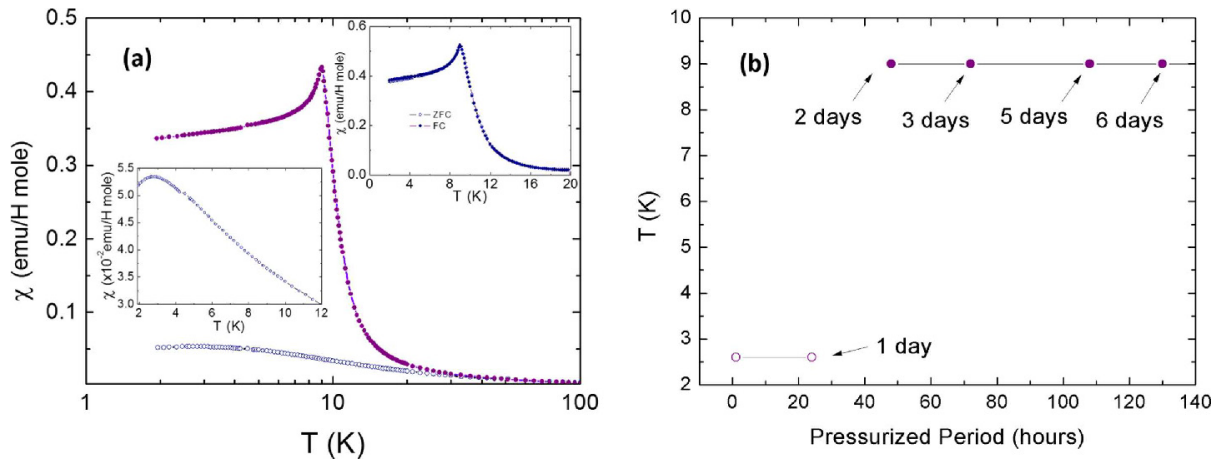

Fig. 6. Susceptibility of pre- and post-pressurized $\mathrm{Cs}_{2} \mathrm{CuCl}_{4}$ with pressing time dependence. (a) The dc susceptibility for prepressurized (open symbol) and post-pressurized (solid symbol) $\mathrm{Cs}_{2} \mathrm{CuCl}_{4}$ with external magnetic field parallel to ac plane under ambient pressure. The susceptibility of the post-pressurized sample shows a transition temperature of $T_{\mathrm{s}}=9 \mathrm{~K}$. The upper inset shows that there is no difference between the zero-field-cool and field-cool susceptibilities. (b) The new ground magnetic phase of post-pressurized $\mathrm{Cs}_{2} \mathrm{CuCl}_{4}$ could be observed under ambient pressure when it was pressed for more than 2 days. Both (a) and (b) from [2].

Table 1. The comparisons for crystal structures between the pre- and post-pressurized $\mathrm{Cs}_{2} \mathrm{CuCl}_{4}$ single crystals at room temperature. From [2].

\begin{tabular}{llll}
\hline & $\begin{array}{l}\text { Pre-pressurized (i) } \\
\mathrm{Cs}_{2} \mathrm{CuCl}_{4}\end{array}$ & $\begin{array}{l}\text { Post-pressurized (ii) } \\
\mathrm{Cs}_{2} \mathrm{CuCl}_{4}\end{array}$ & $\begin{array}{l}\text { Difference between (ii) } \\
\text { and (i) }\end{array}$ \\
\hline$a(\AA)$ & $9.7644(2)$ & $9.7663(4)$ & 0.0019 \\
$b(\AA)$ & $7.6143(3)$ & $7.6094(3)$ & -0.0049 \\
$c(\AA)$ & $12.3988(5)$ & $12.4146(5)$ & 0.0158 \\
$V\left(\AA^{3}\right)$ & $921.84(5)$ & $922.6(6)$ & 0.76 \\
$\mathrm{Cu}(1)-\mathrm{Cu}(2)(\AA)$ & $7.6143(3)$ & $7.6094(3)$ & -0.0049 \\
$\mathrm{Cu}(1)-\mathrm{Cu}(3)(\AA)$ & $7.2851(1)$ & $7.2804(6)$ & 0.0053 \\
\hline
\end{tabular}

with a transition temperature of $9 \mathrm{~K}$. We have observed no significant difference in the crystal structure between the post-pressurisation sample with new magnetic ground state and the uncompressed one with antiferromagnetic quantum spin liquid phase. Geometrically frustrated spin structures have been the focus of great attention from a broad range of the scientific community due to the potential functional and fundamentally interesting physical properties they display such as superconductivity, magnetism and ferroelectricity [31-36]. The physical properties result from the competition between exchange coupling strengths on the frustrated antiferromagnetic spin lattice. The magnetic phases and phase transitions of frustrated antiferromagnetic quantum spin systems have been investigated experimentally and theoretically $[37,38]$. The new phase displays magnetic properties that are similar to chiral helimagnet-like behaviour in stark contrast to the quantum spin liquid phase of unpressurised $\mathrm{Cs}_{2} \mathrm{CuCl}_{4}$.

$\mathrm{Cs}_{2} \mathrm{CuCl}_{4}$ has been shown to be a two-dimensional quantum spin-liquid phase [39], by neutron scattering [40-42], magnetization [43] and specific heat [44] measurements. The crystal structure of $\mathrm{Cs}_{2} \mathrm{CuCl}_{4}$ is orthorhombic with space group Pnma [45]. It is composed of $\mathrm{CuCl}_{4}^{2}-$ tetrahedra and $\mathrm{Cs}^{+}$ions as shown in Figure 5. The magnetic structure can be thought of as anisotropic spin-1/2 triangular antiferromagnet, with $\mathrm{Cu}^{2+}$ ions on a geometrical bc plane. The interchain exchange coupling J'
$(=0.125 \mathrm{meV})$ between $\mathrm{Cu}^{2+}$ ions along zigzag directions on the triangular bc plane is weaker than the intrachain exchange coupling $\mathrm{J}(=0.375 \mathrm{meV})$ along $b$-axis, with the coupling ratio of $\mathrm{J} / \mathrm{J}=0.34$ [39-41].

There are two striking things about this pressure induced magnetic state. (1) The nature of the magnetic state is very different from the ambient pressure ground state despite minimal distortions of the crystal structure. See Figure 6a. (2) The transition appears to take a very long time to take place see Figure $6 \mathrm{~b}$.

Table 1 shows that the new ground state is certainly not the result of any dramatic collapse or restructuring of the lattice (Fig. 6). A compression of the $c$-axis is the biggest change and this is of the order of $0.1 \%$.

Figure 7 sums up the nature of the new magnetic ground state. The magnitude of the magnetisation has been increased by up to two orders of magnitude at the lowest temperatures. Just as strikingly the field dependence of the new state is in stark contrast to the largely field independent behaviour displayed by the unpressurised $\mathrm{Cs}_{2} \mathrm{CuCl}_{4}$ over the same field range. This can be seen clearly in the field dependence of the temperature of the peak in the magnetisation, $T_{\mathrm{s}}$ shown in Figure $7 \mathrm{~b}$. As this material is driven closer to metallisation with the application of higher pressure we may expect further transitions as the degeneracy of the may related ground states is lifted. 

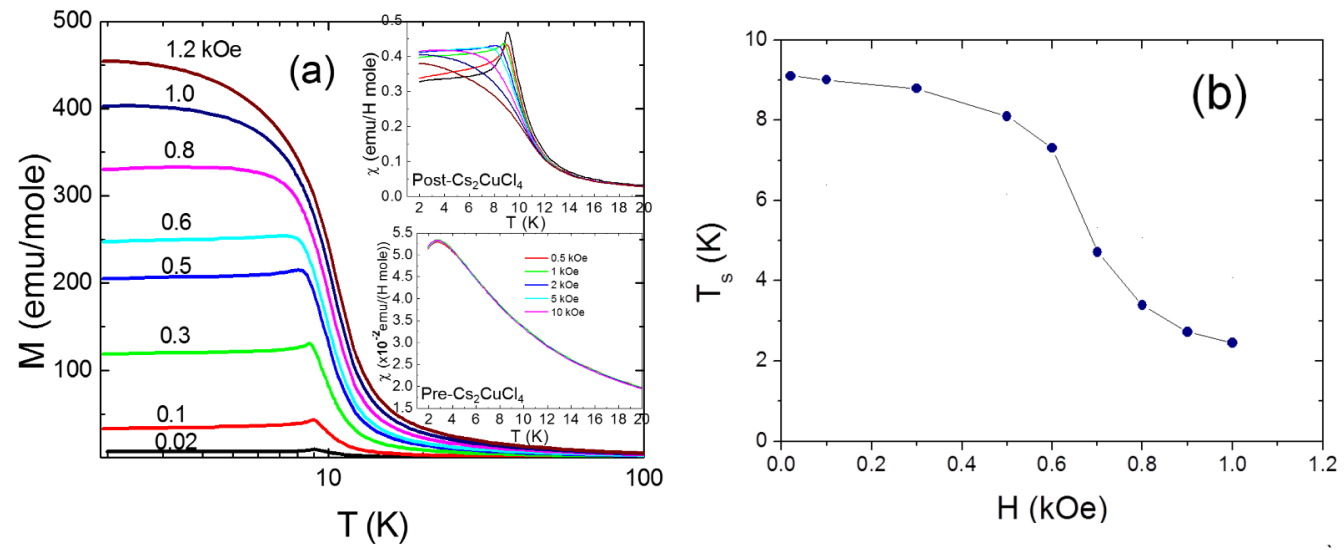

Fig. 7. Magnetization of the post-pressurized $\mathrm{Cs}_{2} \mathrm{CuCl}_{4}$. Temperature dependence of magnetisation curves of the postpressurized $\mathrm{Cs}_{2} \mathrm{CuCl}_{4}$ with each field (parallel to ac plane) from 0.02 to $1.2 \mathrm{kOe}$ under ambient pressure in the main figure (a). The transition temperature is about $9 \mathrm{~K}$ up to $0.1 \mathrm{kOe}$. But as field increases, the peak transition temperature, $T_{\mathrm{s}}$, is decreased and, above $1 \mathrm{kOe}$, has disappeared (b). The upper inset in figure (a) shows the susceptibility from the magnetisation curve normalized by each field for the post-pressurized $\mathrm{Cs}_{2} \mathrm{CuCl}_{4}$. The lower inset in figure (a) shows the susceptibility of the pre-pressurized $\mathrm{Cs}_{2} \mathrm{CuCl}_{4}$ without change of transition temperature for several magnetic fields. Both (a) and (b) from [2].

\section{Conclusions}

Having followed the philosophy of using the appropriate tuning parameter to explore the phase space of potentially highly degenerate systems we have discovered several new phase transitions in the magnetic insulators $\mathrm{FePS}_{3}$ and $\mathrm{Cs}_{2} \mathrm{CuCl}_{4}$. The proximity to long range order remains one of the most productive regions of phase space for the discovery on novel states and phenomena. The possibilities for coupled or competing order parameters is enormous - multicriticality is a very active area for both theory and experiment. Reduced dimensionality has been shown to be helpful for increasing the energy scales at which novel phenomena may emerge. This has been experiment led and looks likely to continue to be so. It should be no surprise that proximity to a quantum phase transition in general and a quantum critical point in particular leads to many new discoveries: this has already been observed in metallic systems. It is exciting, however, that the same motivation and conceptual methodology can be equally applied to magnetic insulators. In fact by starting from magnetic insulators and then metallising through the application of pressure we can only expect to find a strongly correlated metallic state. As we continue towards the initial aim of discovering and describing the metallic state in these metallised quasi-two dimensional systems we anticipate many other interesting new states and phenomena will present themselves.

A further lesson can be gleaned. Although the band gap in many insulators is large and the pressure needs to close it may be high - in systems where the gap comes from strongly correlated electrons and/or the presence of a Van der Waals gap moderate pressure is a good tuning parameter. Indeed there may be other states that are particularly susceptible to applied pressure or strain.

Much of this article is based on work in two recent publications and therefore the authors wish to acknowledge Matthew
Coak, Andrew Wildes, Hayrullo Hamidov, Cheng Liu, David Jarvis, Paul Nahai-Williamson and Dominik Daisenberger for discussions, help with figures and more on the $\mathrm{FePS}_{3}$ work, and Hyeong-Jin Kim, Cheng Liu, Sae Hwan Chun, Kee Hoon Kim, H.T. Yi and Sang-Wook Cheong for their work on $\mathrm{Cs}_{2} \mathrm{CuCl}_{4}$. We acknowledge support from KAZATOMPROM Kazakhstan, EPSRC and Jesus College, Cambridge. The work at SNU was supported by National Creative Research Initiative (2010-0018300). Work at Rutgers is funded by the Gordon and Betty Moore Foundation's EPiQS Initiative through Grant GBMF4413 to the Rutgers Center for Emergent Materials. We thank CamCool Research Ltd. for providing the pressure cells used in this work. The high pressure XRD work on $\mathrm{FePS}_{3}$ was carried out with the support of the Diamond Light Source and we thank Heribert Wilhelm for advice and help with the X-ray diffraction experiments at Diamond. We would also like to acknowledge support the CHT Uzbekistan programme. The work was carried out with financial support from the Ministry of Education and Science of the Russian Federation in the framework of Increase Competitiveness Program of NUST MISiS (K 2-2017-024). This work was supported by IBS-R009-G1.

\section{Author contribution statement}

Both authors contributed equally to the preparation of this paper.

Open Access This is an open access article distributed under the terms of the Creative Commons Attribution License (http://creativecommons.org/licenses/by/4.0), which permits unrestricted use, distribution, and reproduction in any medium, provided the original work is properly cited.

\section{References}

1. Y. Song, Z. Yamani, C. Cao, Y. Li, C. Zhang, J.S. Chen, Q. Huang, H. Wu, J. Tao, Y. Zhu, W. Tian, S. Chi, H. Cao, 
Y. Huang, M. Dantz, T. Schmitt, R. Yu, A. Nevidomskyy, E. Morosan, P. Dai, Nat. Commun. 7, 13879 (2016)

2. H.-J. Kim, C.R.S. Haines, C. Liu, S.H. Chun, K.H. Kim, H.T. Yi, S.-W. Cheong, S.S. Saxena, Low Temp. Phys. 43, 901 (2017)

3. S.E. Rowley, L.J. Spalek, R.P. Smith, M.P.M. Dean, M. Itoh, J.F. Scott, G.G. Lonzarich, S.S. Saxena, Nat. Phys. 10, 367 (2014)

4. P. Monthoux, G.G. Lonzarich, Phys. Rev. B 66, 224504 (2002)

5. W. Klingen, G. Eulenberger, H. Hahn, Die Naturwissenschaften 55, 229 (1968)

6. G. Ouvrard, R. Brec, J. Rouxel, Mater. Res. Bull. 20, 1181 (1985)

7. R. Brec, D.M. Schleich, G. Ouvrard, A. Louisy, J. Rouxel, Inorg. Chem. 18, 1814 (1979)

8. K.C. Rule, G.J. McIntyre, S.J. Kennedy, T.J Hicks, Phys. Rev. B 76, 134402 (2007)

9. S. Kurosawa, K. Saito, Y. Yamaguchi, J. Phys. Soc. Jpn 52, 3919 (1983)

10. K. Ichimura, M. Sano, Synth. Met. 45, 203 (1991)

11. P.A. Joy, S. Vasudevan, Phys. Rev. B 465425 (1992)

12. G. Le Flem, R. Brec, G. Ouvard, A. Louisy, P. Segransan, J. Phys. Chem. Solids 43, 455 (1982)

13. J. Rouxel, P. Molinie, L.H. Top, J. Power Sources 9, 345 (1983)

14. N. Kurita, K. Nakao, J. Phys. Soc. Jpn 58, 610 (1989)

15. N. Kurita, K. Nakao, J. Phys. Soc. Jpn 58, 232 (1989)

16. V. Grasso, L. Silipigni, Rivista Del Nuovo Cimento 25, 1 (2002)

17. D. Lançon, H.C. Walker, E. Ressouche, B. Ouladdiaf, K.C. Rule, G.J. McIntyre, T.J. Hicks, H.M. Rønnow, A.R. Wildes, Phys. Rev. B 94, 214407 (2016)

18. A.R. Wildes et al., Phys. Rev. B 92, 224408 (2015)

19. A.R. Wildes, K.C. Rule, R.I. Bewley, M. Enderle, T.J. Hicks, J. Phys.: Condens. Matter, 24, 416004 (2012)

20. A.R. Wildes, H.M. Rønnow, B. Roessli, M.J. Harris, K.W. Godfre, Phys. Rev. B 74, 094422 (2006)

21. A.R. Wildes, B. Roessli, B. Lebech, K.W. Godfrey, J. Phys.: Condens. Matter, 10, 6417 (1998)

22. J.U. Lee, S.M. Lee, J.H. Ryoo, S.M. Kang, T.Y. Kim, P.W. Kim, C.H. Park, J.G. Park, H.S. Cheong, Nano Lett. 16, 7433 (2016)

23. V. Grasso, S. Santangelo, M. Piacentini, Solid State Ion. 20, 9 (1986)

24. M.H. Whangbo, R. Brec, G. Ouvrard, J. Rouxel, Inorg. Chem. 24, 2459 (1985)
25. V. Zhukov, S. Alvarez, D. Novikov, J. Phys. Chem. Solids 57, 647 (1996)

26. M. Piacentini, F.S. Khumalo, C.G. Olson, J.W. Anderegg, D.W. Lynch, Chem. Phys. 65, 289 (1982)

27. C.R.S. Haines, M.J. Coak, P. Nahai-Williamson, C. Liu, S.S. Saxena, Pressure induced insulator-to-metal transition in $\mathrm{FePS}_{3}$, in SCES2014 (2014), number We-217, p. 788

28. M. Tsurubayashi, K. Kodama, M. Kano, K. Ishigaki, Y. Uwatoko, T. Watanabe, K. Takase, Y. Takano, Pressure dependence of electric resistivity of $\mathrm{FePS}_{3}$, Nihon University Science and Engineering Office, Academic Lecture Abstract Collection

29. C.R.S. Haines, M.J. Coak, G.I. Lampronti, C. Liu, H. Hamidov, A.R. Wildes, D. Daisenberger, P. Nahai-Williamson, S.S. Saxena, Structural and electronic phase transitions in $\mathrm{FePS}_{3}$ under the application of pressure, ArXiv:1801.10089 (2018)

30. K. Momma, F. Izumi, J. Appl. Cryst. 44, 1272 (2011)

31. S.-W. Cheong, M. Mostovoy, Nat. Mater. 6, 13 (2007)

32. T. Katsufuji, H. Takagi, Phys. Rev. B 64, 054415 (2001)

33. M. Bruhwiler, S.M. Kazakov, N.D. Zhigadlo, J. Karpinski, B. Batlogg, Phys. Rev. B 70, 020503 (2004)

34. H. Kawamura, J. Phys.: Condens. Matter 10, 4707 (1998)

35. J.S. Gardner, M.J.P. Gingras, J.E. Greedan, Rev. Mod. Phys. 82, 53 (2010)

36. B.J. Powell, R.H. McKenzie, Rep. Prog. Phys. 74, R827 (2011)

37. H.T. Diep (Ed.), Frustrated Spin Systems (WorldScientific, 2004)

38. P.W. Anderson, Mater. Res. Bull. 8, 153 (1973)

39. R. Coldea, D.A. Tennant, A.M. Tsvelik, Z. Tylczynski, Phys. Rev. Lett. 86, 1335 (2001)

40. R. Coldea, D.A. Tennant, R.A. Cowley, D.F. McMorrow, B. Dorner, Z. Tylczynski, J. Phys.: Condens. Matter 8, 7473 (1996)

41. R. Coldea, D.A. Tennant, K. Habicht, P. Smeibidl, C. Wolters, Z. Tylczynski, Phys. Rev. Lett. 88, 137203 (2002)

42. R. Coldea, D.A. Tennant, Z. Tylczynski, Phys. Rev. B 68, $134424(2003)$

43. Y. Tokiwa, T. Radu, R. Coldea, H. Wilhelm, Z. Tylczynski, F. Steglich, Phys. Rev. B 73, 134414 (2006)

44. T. Radu, H. Wilhelm, V. Yushankhai, D. Kovrizhin, R. Coldea, Z. Tylczynski, T. Luhmann, F. Steglich, Phys. Rev. Lett. 95, 127202 (2005)

45. B. Morosin, E.C. Lingafelter, J. Phys. Chem. 65, 50 (1961) 